\title{
Publisher Correction: Autophagy as a promoter of longevity: insights from model organisms
}

\section{Malene Hansen, David C. Rubinsztein (D) and David W. Walker}

Nature Reviews Molecular Cell Biology (2018) https://doi.org/10.1038/s41580-018-0033-y Published online 13 July 2018

In the original article a Note added in proof was not included. This has now been amended. In addition, the sentence highlighting ref. 162 referred to $\mathrm{Bcl}-1$ as a negative regulator of beclin 1 . This was corrected to BCL-2.

https://doi.org/10.1038/s41580-018-0048-4 I Published online 25 July 2018 